\title{
The Effects of Edge Weights on Correlating Dynamical Networks Comparing Unweighted and Weighted Brain Graphs of nervus opticus Patients
}

\author{
Christian Moewes and Rudolf Kruse \\ Faculty of Computer Science, Otto-von-Guericke University, Universitätsplatz 2, 39106 Magdeburg, Germany \\ \{cmoewes, rkruse\}@ovgu.de
}

Keywords: Dynamical Networks, Regression, Vector Autoregression Weighted Graph

\begin{abstract}
We are interested in the regression analysis of dynamical networks. Our goal is to predict real-valued function values from a given observation which is manifested as series of graphs. Every observation is described by a set of dependent variables that we want to predict using the dynamical graphs. These graphs change their edges over time, while the set of nodes is assumed to be constant. Such settings can be found in many real-world applications, e.g., communication networks, brain connectivity, microblogging. We apply several measures to every graph in the series to globally describe its evolution. The resulting multivariate time series is used to learn vector autoregressive (VAR) models. The parameters of these models can be used to correlate them with the dependent variables. The graph measures typically depend on the type of edges, i.e., weighted or unweighted. So do the VAR models and thus the regression results. In this paper we argue that it is beneficial to keep edge weights in this setting. To support this claim, we analyze electroencephalographic (EEG) networks from patients suffering from visual field defects. The edge weights are in the unit interval and might be thresholded. We show that dynamical network models for weighted edges lead to similar regression performances compared to those of unweighted graphs.
\end{abstract}

\section{INTRODUCTION}

Complex networks be constructed from any set of objects that "interact" with each other. So, based on the large number different objects with this property, complex networks can be found in nearly every part of our world, e.g., in social science (Wassermann and Faust, 1994), computer science (Faloutsos et al., 1999), medicine (Pereira-Leal et al., 2004), biology (Fischhoff et al., 2007), neuroscience (Sporns, 2010), and the World Wide Web (Kleinberg et al., 1999). Many researchers in these and other fields think that the analysis of complex networks may be useful to extract their intrinsic regularities, causal relations, and other useful pieces of information.

Probably the most complex network in the universe is the human brain. Since (Varela et al., 2001), if not earlier, it became very common in neuroscience to analyze functional networks. One property of these complex networks is their nature to dynamicallysometimes even chaotically-change their edges over time. They are typically obtained from neuroimaging methods, e.g., electroencephalography (EEG), electrocorticography (ECoG), magnetoencephalography (MEG), or functional magnetic resonance imaging
(fMRI). These methods record the activity of different brain regions - on the skull, on the brain meninges, or even inside the brain.

Whenever two brain regions, i.e., the nodes of the brain graph, are co-active, they are functionally connected to each other. These connections lead to a complex brain network which represents a high-level abstraction of the biological nervous cells. The analysis of these brain networks has already led to a better understanding of the functionality of different brain centers and the brain as a whole (Sporns, 2010).

Most research on complex graphs-no matter if neuroscientific or not-has mainly focused on static graphs. Such a static network is simply obtained by averaging the connections over time. Of course, it is far easier to analyze static networks than a series of networks. Nevertheless averaging diminishes an important property, i.e., the dynamics of such complex networks.

We argue that the dynamical nature of networks can also be exploited to analyze complex networks and some of their functions. Some first findings support this claim, at least for "damaged" human brain webs: In (Moewes et al., 2013) patients' EEG has been correlated to the size of visual field deficits that 
resulted from optic nerve damages (Wüst et al., 2002). The authors identified a relation between the extend of the vision loss and the dynamics of the functional brain connectivity. More precisely, the authors of this paper propose the first model-based approach to capture the relevant features of a dynamical complex network (Moewes et al., 2013).

In the above-mentioned work of the authors, unweighted graphs have been constructed to facilitate the computation of the network models. Therefore some patient-independent threshold for the edge weights had to be identified. The question we address in this paper is whether the tedious and somewhat arbitrary choice can be omitted. We thus claim that it is beneficial to use weighted graphs instead of unweighted ones.

To support this hypothesis, we analyze the same kind of EEG networks used in (Moewes et al., 2013). We apply different thresholds to the weighted edges to obtain an unweighted graph. Eventually we show that dynamical network models for weighted edges lead to similar if not better regression results than those of unweighted graphs. Thus the tedious and somewhat arbitrary choice of an edge weight threshold is superfluous.

The rest of the paper is organized as follows. Section 2 introduces the concept of dynamical networks to describe functional brain connectivity. Section 3 describes the experimental setup we have used to compare the regression performance of weighted and unweighted graphs. The results of this experiment are listed in Section 4. Finally, Section 5 concludes the paper.

\section{DYNAMICAL NETWORK ANALYSIS}

Having defined the functional connectivity of two brain regions, the corresponding dynamical brain network can be obtained. We remark that functional connectivity is just a statistical relationship between brain regions without implying any causal coherence (Pearl, 2009). There exist many ways to compute functional connectivity. We refer the interested reader to (Wendling et al., 2009) for a review on EEG connectivity measures.

\subsection{Synchronization Likelihood}

Out of these methods, we have used the synchronization likelihood (SL) (Stam and van Dijk, 2002) since it has been used in the literature to study the relation- ship between structural network damage and functional connectivity (Stam et al., 2007).

Consider a multivariate time series (e.g. a multichannel EEG recording) of length $N$ with $n$ variables. Let measurement $x_{i, k}$ be observed at timestamp $i$ in channel $k$. For the SL, a time-delay embedding is computed by

$$
X_{i, k}=\left(x_{i, k}, x_{i+L, k}, x_{i+2 \cdot L, k}, \ldots, x_{i+(m-1) \cdot L, k}\right)
$$

where $L$ is the lag and $m$ the dimension of the embedding. The state vectors $X_{i, k}$ shall capture the relevant patterns of the signal.

Now consider two channels $A, B$. The probability that $X_{i, k}$ are closer to each other than $\varepsilon$ is

$$
P_{i, k}^{\varepsilon}=\frac{1}{2\left(W_{2}-W_{1}\right)} \sum_{\substack{j \\ W_{1}<|i-j|<W_{2}}}^{N} \theta\left(\varepsilon-d\left(X_{i, k}, X_{j, k}\right)\right)
$$

where $d$ is the Euclidean distance (or any other distance measure). For each $k$ and $i$, a critical distance $\varepsilon_{i, k}$ is computed such that $P_{i, k}^{\varepsilon_{i, k}}=p_{\text {ref }}$ whereas $p_{\text {ref }} \ll 1$ is some user-defined threshold. For each pair of points in time $(i, j)$ within $W_{1}<|i-j|<W_{2}$, the number of channels $H_{i, j}$ for which $d\left(X_{i, k}, X_{j, k}\right)<\varepsilon_{i, k}$ is computed by

$$
H_{i, j}=\theta\left(\varepsilon_{i, A}-d\left(X_{i, A}, X_{j, A}\right)\right)+\theta\left(\varepsilon_{i, B}-d\left(X_{i, B}, X_{j, B}\right)\right)
$$

where $\theta(x)=0$ if $x \leq 0$ and $\theta(x)=1$ for $x>0$. Then the synchronization likelihood is then given by

$$
S L_{i}=\frac{1}{2 p_{\mathrm{ref}}\left(W_{2}-W_{1}\right)} \sum_{\substack{j \\ W_{1}<|i-j|<W_{2}}}^{N}\left(H_{i, j}-1\right) .
$$

Now the final brain graph can be computed. Note that only two of these the parameters are needed to compute SL if prior information about the frequency range and temporal resolution of the signal are given (Montez et al., 2006).

\subsection{Graph Measures}

We remark that any functional connectivity measure based on a sliding window will change its value over time. So do the edge weights of the corresponding brain graph. In (Moewes et al., 2013) the authors described these changes by learning how graph measures evolve.

Therefore we consider a set of different graph measures, i.e., the number of cliques, the density, and the squared distance between the current graph and the previous one (Bunke, 1997). 


\subsection{Vector Autoregressive Models}

Vector autoregressive (VAR) models (Lütkepohl, 2005) have been shown to capture a high proportion of variance in these data.

A VAR model with $p$ lags is given by

$$
\vec{x}_{t}=c+\sum_{i=1}^{p} A_{i} \vec{x}_{t-i}+\varepsilon_{t} .
$$

where $c$ is a constant, $A_{i}$ is a matrix storing the relationships between every pair of variable at point $t-i$, and $\varepsilon_{t}$ is white noise. Flattening the coefficients $A_{i}$ to a feature vector is beneficial for statistical learning methods.

\section{EXPERIMENTS}

In our experiments we used EEG data from 33 visually impaired subjects suffering from optic nerve damages (Wüst et al., 2002). For every patient, several optometric tests had been performed to describe the location and the size of the optic nerve damage for both eyes (Sabel et al., 2011). Among them are high resolution perimetry (HRP) (Kasten et al., 1998), static perimetry, kinetic perimetry, and the assessment of visual acuity (Bailey and Lovie, 1976). Based on these tests an expert defined the following clinical variables relevant to quantify the vision loss of both eyes:

- detection accuracy in HRP visual field (\%),

- foveal threshold in static perimetry $(\mathrm{dB})$,

- mean threshold in static perimetry (whole $30^{\circ}$ visual field, dB),

- mean eccentricity in kinetic perimetry $\left(^{\circ}\right)$,

- visual acuity of near vision (LogMAR scale),

- visual acuity of far vision (LogMAR scale).

Nearly every optometric test is very tiring, timeconsuming, and error-prone. Each one of them served as variable assumed to be depended from the dynamics in the EEG. It is our motivation to find good correlates of the EEG signal and these tests. If successful, a good prediction model may determine the size of the optic nerve damage by solely looking at EEG data that can be recorded much faster.

To preprocess the EEG data we did the following steps in EEGLAB (Delorme and Makeig, 2004):

- manually removal of noisy time frames at beginning/end of each recording,

- removal of uncommon EEG channels across all subjects (28 were used),
- high-pass filtering with cutoff frequency at $1 \mathrm{~Hz}$ to remove slow movements,

- notch filtering $50 \mathrm{~Hz}$ to remove the European power line frequency,

- low-pass filtering with cutoff frequency at $95 \mathrm{~Hz}$,

- re-referencing by the average electrode,

- down-sampling to $250 \mathrm{~Hz}$ to reduce the costs of SL computation,

- manual removal of biological artifacts using independent component analysis (Makeig et al., 1996).

Biological artifacts that stem from electromyographic (EMG) or electrocardiograph (EKG) signal appear as noise in the recorded EEG signal in all variations. To remove EMG and ECG signals ICA was applied to very carefully remove noisy components.

We applied FIR filters to obtain the conventional frequency bands. They are associated with different brain states (Edwards, 2007). These bands are $\delta: f \in(1,4] \mathrm{Hz}, \theta: f \in(4,7] \mathrm{Hz}, \alpha: f \in[8,12] \mathrm{Hz}$, $\beta: f \in[13,30] \mathrm{Hz}$, and $\gamma: f \in[30,50] \mathrm{Hz}$. We expect the optometric variables to explainable by the dynamics of functional connectivity in these frequency bands. Functional connectivity was computed by the synchronization likelihood (Stam and van Dijk, 2002): We used an outer window length of $W_{2}=3 \mathrm{~s}$ and a reference probability of $p_{\text {ref }}=0.02$. To capture most of the dynamics, the sliding window shifted every $0.5 \mathrm{~s}$, i.e. an overlay of $1 / 6$. Note that the analysis of the averaged graphs did not result in any useful model (Held et al., 2012).

We applied the measures mentioned in Section 2 to every brain graph. We used the Python package igraph (Csárdi and Nepusz, 2006) to accomplish this task. This resulted in a multivariate time series for each subject and each frequency band. Every time series was then fitted by a VAR model with $p=1,2$ for simplicity. Eventually we obtained $p \cdot 3 \cdot 3=9$ and 18 parameters, respectively, describing the dynamics of the corresponding multivariate time series.

\section{RESULTS}

We used ordinary least-squares regression and computed its leave-one-out (LOO) estimation of the true error. All models have been fit using the Python packages sklearn (Pedregosa et al., 2011) and statsmodels (Seabold and Perktold, 2010). Both the VAR parameters and the optometric variables have been zscore normalized, i.e., we subtracted the mean and divided by the standard deviation. The regression per- 
Table 1: Mean-squared errors (MSE) of the VAR models to describe the vision loss of the right eye. The results from the weighted networks are shown on the left-hand side, the ones from the unweighted network are shown on the right-hand side.

\begin{tabular}{|c|r|r|r|r|r|}
\hline weighted & $\delta$ & $\theta$ & $\alpha$ & $\beta$ & $\gamma$ \\
\hline HRP_DA & 0.963 & 1.297 & 1.055 & 1.403 & 2.015 \\
\hline SP_FT & 1.335 & 1.447 & 1.330 & 1.142 & 1.869 \\
\hline SP_MT & 1.134 & 1.542 & 0.897 & 1.436 & 2.087 \\
\hline KP_ME & 1.259 & 1.559 & 1.369 & 1.354 & 1.844 \\
\hline VA_N_log & 1.235 & 1.229 & 1.385 & 1.313 & 1.532 \\
\hline VA_F_log & 1.209 & 1.283 & 1.348 & 1.254 & 1.431 \\
\hline
\end{tabular}

\begin{tabular}{|c|r|r|r|r|r|}
\hline$S L \geq 0.5$ & $\delta$ & $\theta$ & $\alpha$ & $\beta$ & $\gamma$ \\
\hline HRP_DA & 1.337 & 1.291 & 1.448 & 1.204 & 2.440 \\
\hline SP_FT & 1.202 & 0.941 & 1.571 & 2.159 & 1.627 \\
\hline SP_MT & 1.197 & 1.140 & 1.396 & 2.261 & 1.949 \\
\hline KP_ME & 1.318 & 1.601 & 1.435 & 2.329 & 1.245 \\
\hline VA_N_log & 1.215 & 1.365 & 1.483 & 2.115 & 1.779 \\
\hline VA_F_log & 1.181 & 1.411 & 1.580 & 2.656 & 1.411 \\
\hline
\end{tabular}

Table 2: Mean-squared errors (MSE) of the VAR models to describe the vision loss of the right eye. The results from two unweighted networks that used different thresholds are shown on both sides of the table.

\begin{tabular}{|c|r|r|r|r|r|}
\hline$S L \geq 0.7$ & $\delta$ & $\theta$ & $\alpha$ & $\beta$ & $\gamma$ \\
\hline HRP_DA & 1.371 & 1.580 & 1.144 & 1.148 & 0.979 \\
\hline SP_FT & 1.314 & 1.275 & 1.385 & 1.070 & 0.807 \\
\hline SP_MT & 1.336 & 1.546 & 1.282 & 0.857 & 1.055 \\
\hline KP_ME & 1.493 & 1.518 & 1.626 & 0.971 & 1.081 \\
\hline VA_N_log & 1.270 & 1.321 & 1.292 & 1.183 & 0.752 \\
\hline VA_F_log & 1.256 & 1.242 & 1.325 & 1.295 & 0.838 \\
\hline
\end{tabular}

\begin{tabular}{|c|r|r|r|r|r|}
\hline$S L \geq 0.2$ & $\delta$ & $\theta$ & $\alpha$ & $\beta$ & $\gamma$ \\
\hline HRP_DA & 0.963 & 1.485 & 1.290 & 1.422 & 1.187 \\
\hline SP_FT & 1.335 & 1.375 & 1.751 & 0.992 & 1.175 \\
\hline SP_MT & 1.134 & 1.280 & 1.130 & 1.358 & 1.244 \\
\hline KP_ME & 1.259 & 1.459 & 1.476 & 1.575 & 1.428 \\
\hline VA_N_log & 1.235 & 1.143 & 1.959 & 0.733 & 1.136 \\
\hline VA_F_log & 1.209 & 1.169 & 2.199 & 0.758 & 1.014 \\
\hline
\end{tabular}

formance of the normalized data was measured by the mean-squared error (MSE).

Tables 1 and 2 on the next page summarize our analyzes for the right eye. Weighted networks lead to MSE that are competitive to the ones of the thresholded networks. For low-frequency bands (i.e., $\delta$, $\theta, \alpha)$, the weighted networks produce even smaller errors. This is in line with the previous results in (Moewes et al., 2013) where 8 subjects less have been used. Equivalently, the results for the left eye are shown in Tables 3 and 4. They do confirm the findings.

\section{CONCLUSIONS}

We have advocated not to threshold edge weights when analyzing dynamical networks. The choice of a threshold to obtain an unweighted graphs can be omitted if the graphs are used for regression models. To show this we have analyzed EEG networks from patients suffering from visual field defects. We have thresholded the edge weights using several different values. Still, the models describing the weighted dynamical network lead to similar errors compared to those of unweighted graphs.

We plan to enlarge the data set to further increase the quality of our models. We also want to establish a way to combine the optometric tests of both eyes. A comparison of brain networks to classical EEG representations is in preparation. Furthermore we are working on the creation of different data sets, e.g., communication networks and microblogs, to general- ize our findings.

\section{ACKNOWLEDGMENTS}

The first author thanks Carolin Gall and her students from the Medical Faculty for collecting the EEG data. We give thanks to Hermann Hinrichs from the Medical Faculty for pointing out several hints to preprocess the EEG data. Last not least we thank Bernhard A. Sabel and Michał Bola for fruitful discussions while preparing this paper. 
Table 3: Mean-squared errors (MSE) of the VAR models to describe the vision loss of the left eye. The results from the weighted networks are shown on the left-hand side, the ones from the unweighted network are shown on the right-hand side.

\begin{tabular}{|c|c|c|c|c|c|c|c|c|c|c|c|}
\hline weighted & $\delta$ & $\theta$ & $\alpha$ & $\beta$ & $\gamma$ & $S L \geq 0.5$ & $\delta$ & $\theta$ & $\alpha$ & $\beta$ & $\gamma$ \\
\hline HRP_DA & 1.580 & 1.572 & 1.450 & 1.629 & 1.397 & HRP_DA & 1.164 & 0.760 & 1.398 & 1.217 & 1.054 \\
\hline SP_FT & 1.511 & 1.239 & 1.089 & 1.873 & 1.647 & SP_FT & 1.490 & 1.178 & 1.253 & 1.368 & 1.434 \\
\hline SP_MT & 1.259 & 1.559 & 1.369 & 1.354 & 1.844 & SP_MT & 1.080 & 1.021 & 1.065 & 1.109 & 1.189 \\
\hline KP_ME & 1.534 & 1.103 & 1.305 & 1.456 & 1.471 & KP_ME & 1.286 & 1.011 & 1.221 & 1.047 & 1.222 \\
\hline VA_N_log & 1.541 & 1.140 & 1.298 & 2.193 & 1.578 & VA_N_log & 1.568 & 1.064 & 1.299 & 1.328 & 1.347 \\
\hline VA_F_log & 1.425 & 1.117 & 1.433 & 1.913 & 1.682 & VA_F_log & 1.551 & 1.465 & 1.412 & 1.527 & 1.359 \\
\hline
\end{tabular}

Table 4: Mean-squared errors (MSE) of the VAR models to describe the vision loss of the left eye. The results from two unweighted networks that used different thresholds are shown on both sides of the table.

\begin{tabular}{|c|r|r|r|r|r|}
\hline$S L \geq 0.7$ & $\delta$ & $\theta$ & $\alpha$ & $\beta$ & $\gamma$ \\
\hline HRP_DA & 1.357 & 1.259 & 1.401 & 0.665 & 3.353 \\
\hline SP_FT & 1.423 & 1.524 & 1.283 & 1.698 & 1.412 \\
\hline SP_MT & 1.230 & 1.267 & 1.122 & 1.502 & 1.056 \\
\hline KP_ME & 1.283 & 0.987 & 1.324 & 1.037 & 0.992 \\
\hline VA_N_log & 1.484 & 1.173 & 1.413 & 1.520 & 1.391 \\
\hline VA_F_log & 1.454 & 1.325 & 1.387 & 1.414 & 1.378 \\
\hline
\end{tabular}

\begin{tabular}{|c|r|r|r|r|r|}
\hline$S L \geq 0.2$ & $\delta$ & $\theta$ & $\alpha$ & $\beta$ & $\gamma$ \\
\hline HRP_DA & 1.580 & 0.967 & 1.174 & 1.755 & 1.783 \\
\hline SP_FT & 1.511 & 1.128 & 1.451 & 1.166 & 1.234 \\
\hline SP_MT & 1.291 & 0.931 & 0.745 & 1.344 & 1.367 \\
\hline KP_ME & 1.534 & 1.106 & 1.471 & 1.651 & 1.299 \\
\hline VA_N_log & 1.541 & 1.091 & 1.726 & 1.367 & 1.124 \\
\hline VA_F_log & 1.425 & 1.257 & 1.717 & 1.340 & 1.240 \\
\hline
\end{tabular}

\section{REFERENCES}

Bailey, I. L. and Lovie, J. E. (1976). New design principles for visual acuity letter charts. Am J Optom Physiol Opt, 53(11):740-745.

Bunke, H. (1997). On a relation between graph edit distance and maximum common subgraph. Pattern Recognit Lett, 18(8):689-694.

Csárdi, G. and Nepusz, T. (2006). The igraph software package for complex network research. InterJournal Complex Systems, 1695.

Delorme, A. and Makeig, S. (2004). EEGLAB: an open source toolbox for analysis of single-trial EEG dynamics including independent component analysis. $J$ Neurosci Methods, 134(1):9-21.

Edwards, E. (2007). Electrocortical Activation and Human Brain Mapping. $\mathrm{PhD}$ thesis, University of California, Berkeley, CA, USA.

Faloutsos, M., Faloutsos, P., and Faloutsos, C. (1999). On power-law relationships of the internet topology. In Proceedings of the conference on Applications, technologies, architectures, and protocols for computer communication, SIGCOMM '99, pages 251262, New York, NY, USA. ACM.

Fischhoff, I. R., Sundaresan, S. R., Cordingley, J., Larkin, H. M., Sellier, M., and Rubenstein, D. I. (2007). Social relationships and reproductive state influence leadership roles in movements of plains zebra, equus burchellii. Anim Behav, 73(5):825-831.

Held, P., Moewes, C., Braune, C., Kruse, R., and Sabel, B. A. (2012). Advanced analysis of dynamic graphs in social and neural networks. In Borgelt, C., Gil, M. Á., Sousa, J. M. C., and Verleysen, M., editors, Towards Advanced Data Analysis by Combining Soft Computing and Statistics, pages 205-222. Springer-Verlag.

Kasten, E., WÃijst, S., Behrens-Baumann, W., and Sabel,

B. A. (1998). Computer-based training for the treatment of partial blindness. Nat Med, 4(9):1083-1087.

Kleinberg, J. M., Kumar, R., Raghavan, P., Rajagopalan, S., and Tomkins, A. S. (1999). The web as a graph: measurements, models, and methods. In Proceedings of the 5th annual international conference on Computing and combinatorics, COCOON'99, pages 1-17, Berlin, Heidelberg. Springer-Verlag.

Lütkepohl, H. (2005). New Introduction to Multiple Time Series Analysis. Econometrics / Statistics. SpringerVerlag, Berlin / Heidelberg, Germany.

Makeig, S., Bell, A. J., Jung, T., and Sejnowski, T. J. (1996). Independent component analysis of electroencephalographic data. In Touretzky, D. S., Mozer, M. C., and Hasselmo, M. E., editors, Advances in Neural Information Processing Systems, volume 8, pages 145151, Cambridge, MA, USA. MIT Press.

Moewes, C., Kruse, R., and Sabel, B. A. (2013). Analysis of dynamic brain networks using var models. In Kruse, R., Berthold, M. R., Moewes, C., Gil, M. Á., Grzegorzewski, P., and Hryniewicz, O., editors, Synergies of Soft Computing and Statistics for Intelligent Data Analysis, pages 525-532. Proc. of the 6th Int'l Conf. on Soft Methods in Probability and Statistics (SMPS2012), 4-6 Oct. 2012, Konstanz, Germany.

Montez, T., Linkenkaer-Hansen, K., van Dijk, B. W., and Stam, C. J. (2006). Synchronization likelihood with explicit time-frequency priors. Neuroimage, 33(4):1117-1125.

Pearl, J. (2009). Causal inference in statistics: An overview. Stat Surv, 3:96-146.

Pedregosa, F., Varoquaux, G., Gramfort, and et al. (2011). Scikit-learn: Machine learning in python. JMLR, 12:2825-2830

Pereira-Leal, J. B., Enright, A. J., and Ouzounis, C. A. (2004). Detection of functional modules from protein 
interaction networks. Proteins: Struct Funct Bioinf, 54(1):49-57.

Sabel, B. A., Fedorov, A. B., Naue, N., Borrmann, A., Herrmann, C., and Gall, C. (2011). Non-invasive alternating current stimulation improves vision in optic neuropathy. Restor Neurol Neurosci, 29(6):493-505.

Seabold, S. and Perktold, J. (2010). Statsmodels: Econometric and statistical modeling with python. In van der Walt, S. and Millman, J., editors, Proceedings of the 9th Python in Science Conference (SciPy 2010), pages $57-61$.

Sporns, O. (2010). Networks of the Brain. MIT Press, Cambridge, MA, USA.

Stam, C., Jones, B., Nolte, G., Breakspear, M., and Scheltens, P. (2007). Small-World networks and functional connectivity in alzheimer's disease. Cerebral Cortex, 17(1):92-99.

Stam, C. J. and van Dijk, B. W. (2002). Synchronization likelihood: an unbiased measure of generalized synchronization in multivariate data sets. J Phys D: Nonlinear Phenom, 163(3-4):236-251.

Varela, F., Lachaux, J., Rodriguez, E., and Martinerie, J. (2001). The brainweb: phase synchronization and large-scale integration. Nat Rev Neurosci, 2(4):229239.

Wassermann, S. and Faust, K. (1994). Social Network Analysis: Methods and Applications, volume 8 of Stuctural Analysis in the Social Sciences. Cambridge University Press, Cambridge, UK.

Wendling, F., Ansari-Asl, K., Bartolomei, F., and Senhadji, L. (2009). From EEG signals to brain connectivity: A model-based evaluation of interdependence measures. J Neurosci Methods, 183(1):9-18.

Wüst, S., Kasten, E., and Sabel, B. A. (2002). Blindsight after optic nerve injury indicates functionality of spared fibers. J Cogn Neurosci, 14(2):243-253. 